\title{
Motion Reliability Modeling and Evaluation for Manipulator Path Planning Task
}

\author{
Tong Li, Qingxuan Jia, Gang Chen, and Hanxu Sun \\ School of Automation, Beijing University of Posts and Telecommunications, Beijing 100876, China \\ Correspondence should be addressed to Tong Li; bupt.litong@gmail.com
}

Received 11 October 2014; Accepted 2 March 2015

Academic Editor: Zhan Shu

Copyright ( 2015 Tong Li et al. This is an open access article distributed under the Creative Commons Attribution License, which permits unrestricted use, distribution, and reproduction in any medium, provided the original work is properly cited.

\begin{abstract}
Motion reliability as a criterion can reflect the accuracy of manipulator in completing operations. Since path planning task takes a significant role in operations of manipulator, the motion reliability evaluation of path planning task is discussed in the paper. First, a modeling method for motion reliability is proposed by taking factors related to position accuracy of manipulator into account. In the model, multidimensional integral for PDF is carried out to calculate motion reliability. Considering the complex of multidimensional integral, the approach of equivalent extreme value is introduced, with which multidimensional integral is converted into one dimensional integral for convenient calculation. Then a method based on the maximum entropy principle is proposed for model calculation. With the method, the PDF can be obtained efficiently at the state of maximum entropy. As a result, the evaluation of motion reliability can be achieved by one dimensional integral for PDF. Simulations on a particular path planning task are carried out, with which the feasibility and effectiveness of the proposed methods are verified. In addition, the modeling method which takes the factors related to position accuracy into account can represent the contributions of these factors to motion reliability. And the model calculation method can achieve motion reliability evaluation with high precision and efficiency.
\end{abstract}

\section{Introduction}

As a kind of complicated multichain structure, manipulator can achieve various operations and has good environmental adaptability, which makes it widely used in industrial manufacturing, medical and aerospace fields, and so forth. In order to guarantee the positioning accuracy requirement of various tasks and complicated environment, the motion safety and reliability of manipulator attract extensive attention [1-4]. As a result, motion reliability [5-9] which concentrates on the motion accuracy is used to give a quantitative description for the motion performance of manipulator. Generally, the motion reliability is defined as the probability that the position guarantees the accuracy requirement under the affection of various factors [10]. Since manipulator achieves various tasks via positioning operation, the operation status of manipulator can be reflected in numerical by evaluation of motion reliability.

In order to evaluate motion reliability, the model of motion reliability should be established firstly, during which factors related to position accuracy should be considered. Firstly, the relationship between factors and position accuracy should be derived. Zhuang et al. [11] and Chen et al. [12] established the relationship between position error and parameters deviation of manipulator, respectively. $\mathrm{Wu}[13$, 14] analyzed uncertain factors influencing the mechanical positioning accuracy with interval method. The methods mentioned above can be referenced in establishing motion reliability model. Pandey and Zhang [15] discussed the motion reliability of manipulator considering the clearance in joint independently based on probability theory. The clearance is transformed into errors of joint angles to establish the relationship with motion reliability. However, single factor considered is not enough for the modeling of motion reliability. Rao and Bhatti [16] evaluated the kinematics reliability and dynamics reliability of a two-link structure. Unfortunately, factors related to motion reliability were not taken into account. Moreover, the reliability evaluation for manipulator is more complicated than two-link structure, which needs further discussion. 
Based on the analysis above, factors related to motion reliability are not given enough attention. And a universal model which reflects the relationship between factors and motion reliability is not achieved. The model of motion reliability should take the contribution of factors to position accuracy into account. Actually, factors related to position accuracy are various, such as clearance [17], friction [18], and wear [19]. Many of them are unobservable and uncontrollable and even have coupling relationship between each other, which makes it difficult to establish a model including all factors. In order to reflect the influence on position accuracy caused by clearance, friction, wear, and so forth into model, kinematics parameters and joint angles can be taken as intermediate variables to deliver the influence, which has been implemented in [15]. In this way, the motion reliability model can be established based on the deviations of kinematics parameters and joint angles.

After the establishment of motion reliability model, the model should be calculated to achieve evaluation. For this purpose, Kim et al. [20] used the first order reliability method (FORM) to calculate reliability. Generally, FORM can only solve reliability problem related to a few performance functions. The performance functions which are established with position accuracy related to various factors are too many for FORM to be applied. Kumar et al. [21] and Sharma et al. [22] used genetic algorithms and fuzzy methodology to analyze the reliability, respectively. Dashuang et al. [23] used the traditional Monte Carlo method to calculate the dynamic precision reliability of six degrees of freedom (DOF) mechanism. And the authors [24] have analyzed the motion reliability of a 8-DOF modular robot with Monte Carlo method based on a simple model. However, the recursive relationship between factors and motion reliability is not systematically derived in the model. And too many samples are needed in motion reliability calculation with Monte Carlo method. Actually, the fuzzy methodology and Monte Carlo method are quite complex, which leads to high computation cost during reliability computation. Novi Inverardi and Tagliani [25] calculated the probability density function with the principle of maximum entropy, which greatly decreased the samples in computation compared with Monte Carlo method. The method with maximum entropy shows a new way to achieve motion reliability calculation with high efficiency. Considering the practical application, a simple and feasible method for calculating motion reliability model with high precision should be proposed.

Since many tasks depend on the positioning operation of manipulator, path planning [26] takes an important role in achieving various operations. The paper aims at establishing the motion reliability models of path planning task and achieving evaluation. The motion reliability model of path planning task represents the probability that the position of manipulator guarantees the accuracy threshold during the task. The modeling method proposed in the paper takes the contributions of various factors to position accuracy into account. And, in order to reflect the influence of various factors, kinematics parameters and joint angles are taken as intermediate variables to deliver the influence. As a basis, the motion reliability model can be established based on the devotions of kinematics parameters and joint angles. Considering the complex of multidimensional integrals in the model of continuous trajectory tracking, the approach of equivalent extreme value is introduced to achieve model simplification. As a result, multidimensional integrals for PDF are converted into one dimensional integral. Since huge samples and computation time are needed in traditional method for PDF calculation, a method based on the maximum entropy principle is proposed to achieve calculation with high precision and efficiency. So far, the evaluation of motion reliability can be achieved, and an intuitionistic expression for the performance of manipulator can be obtained with the evaluation result. Meanwhile, calibration and control can be devoted to improving the motion reliability of manipulator for further discussion.

In conclusion, the paper is organized as follow. In Section 2, the mathematical relationship between motion reliability and position accuracy is firstly derived. The influence of the factors related to the position accuracy is delivered to the deviations of kinematics parameters and joint angles, based on which the motion reliability models of path planning task are established. In Section 3, the approach of equivalent extreme value is introduced to simplify the motion reliability model, and method based on the maximum entropy principle is proposed to achieve model calculation. In Section 4, simulations are carried out to verify the effectiveness and correctness of the proposed modeling and evaluation method. And strategies for improving the motion reliability are discussed further based on the evaluation result. The last part is summary.

\section{Motion Reliability Models of Path Planning Task}

Path planning tasks are always divided into point-to-point path planning and continuous trajectory tracking, and their motion reliability models have different focuses. For the model of continuous trajectory tracking, position accuracy of the entire trajectory should be considered, while, for the model of point-to-point path planning, position accuracy of target point is paid more attention. Aiming at the two kinds of path planning, motion reliability models are established based on the analysis of factors related to position accuracy.

\subsection{The Mathematical Expression for Motion Reliability of} Path Planning. The motion reliability for a single trajectory point is discussed. Set position accuracy threshold (namely, the maximum acceptable value of the deviation between the actual and desired position) as $l$ at each direction of the point. Then an enveloping space $\Omega$ is formed as a sphere. The desired position is set as center and $l$ is set as radius. As a result, the Cartesian space at the end-effector can be divided into three subspaces shown in Figure 1. The actual position locating in the sphere is considered to be motion reliable, while it is considered to be motion unreliable when actual position locates outside the sphere. The actual positions locating on the shell are critical positions, which can either be reliable or unreliable at the next movement. Based on the analysis 


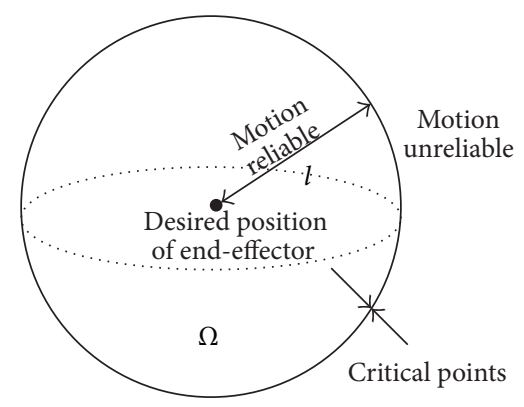

FIGURE 1: The division of the Cartesian space.

above, the mathematical expression when path planning task is motion reliable can be achieved.

(a) Point-to-Point Path Planning. $X^{A}(\Theta)$ and $X^{N}(\Theta)$ represent the actual and desired values of the target point, respectively. $\Theta$ represents factors related to position accuracy. $l_{p}$ represents the position accuracy threshold; then the expression when point-to-point path planning task is motion reliable can be expressed as

$$
R=\operatorname{Pr}\left\{\left|X^{A}(\Theta)-X^{N}(\Theta)\right|<l_{p}\right\} .
$$

Suppose $p(x)$ as the PDF related to position accuracy of the target point, and make $X(\Theta)=\left|X^{A}(\Theta)-X^{N}(\Theta)\right|$; then (1) can be turned into

$$
\operatorname{Pr}\left\{X(\Theta)<l_{p}\right\}=\int_{0}^{l_{p}} p(x) d x
$$

(b) Continuous Trajectory Tracking. When continuous trajectory tracking is motion reliable, the entire trajectory should guarantee the position accuracy threshold. Define $X_{i}^{A}(\Theta)$ and $X_{i}^{N}(\Theta)$ as the actual and desired position at point $i$. Assume position accuracy threshold for every trajectory point is the same, which is expressed as $l_{c}$. The number of trajectory points is $m$. Then the mathematical expression when continuous trajectory tracking is motion reliable can be expressed as

$$
R=\operatorname{Pr}\left\{\bigcap_{i=1}^{m \rightarrow \infty}\left(\left|X_{i}^{A}(\Theta)-X_{i}^{N}(\Theta)\right|<l_{c}\right)\right\}
$$

Define the PDF related to the position accuracy of the entire trajectory points as $p\left(x_{1}, x_{2}, \ldots, x_{m}\right)$, and make $X_{i}(\Theta)=\left|X_{i}^{A}(\Theta)-X_{i}^{N}(\Theta)\right|$; then (3) can be turned into

$$
\begin{aligned}
& \operatorname{Pr}\left\{\bigcap_{i=1}^{m \rightarrow \infty}\left(X_{i}(\Theta)<l_{c}\right)\right\} \\
& \quad=\int_{0}^{l_{c}}\left(\cdots\left(\int_{0}^{l_{c}} p\left(x_{1}, x_{2}, \ldots, x_{m}\right) d x_{1}\right) \cdots\right) d x_{m} .
\end{aligned}
$$

2.2. Analysis of Factors Related to Position Accuracy. In (2) and (4), $\Theta$ represents the factors related to position accuracy. In practice, there are too many factors influencing position accuracy, such as clearance, friction, wear, deformation, assembly tolerances, and flexibility. However, many of them are unobservable and uncontrollable; the accurate model between these factors and position accuracy is hard to obtain. Furthermore, not all the factors have direct influence on the position accuracy in Cartesian space. For example, clearance and friction have influence on the accuracy of joint angles, while assembly tolerances and deformation mainly have influence on geometry parameters. Therefore, the influence caused by these factors can be represented by the deviation of kinematics parameters and joint angles, which is considered as intermediate variables to deliver the influence to the position accuracy at the end-effector. Besides, the deviations of kinematics parameters and joint angles are easy to be measured and adjusted. In this way, deviations of $\mathrm{DH}$ parameters are used to form the factor $\Theta$. And motion reliability can be modeled based on the relationship between deviations of $\mathrm{DH}$ parameters and position accuracy.

For $n$-DOF manipulator, the transformation matrix between $(j-1)$ th and $j$ th coordinate system is described based on parameters $\alpha_{j-1}, a_{j-1}, d_{j}, \theta_{j}$ :

$\mathbf{T}_{j}$

$$
\begin{aligned}
& =T_{j}\left(\alpha_{j-1}, a_{j-1}, d_{j}, \theta_{j}\right) \\
& =\left[\begin{array}{cccc}
\cos \theta_{j} & -\sin \theta_{j} \cos \alpha_{j-1} & \sin \theta_{j} \sin \alpha_{j-1} & a_{j-1} \cos \theta_{j} \\
\sin \theta_{j} & \cos \theta_{j} \cos \alpha_{j} & -\cos \theta_{j} \sin \alpha_{j-1} & a_{j-1} \sin \theta_{j} \\
0 & \sin \alpha_{j-1} & \cos \alpha_{j-1} & d_{i} \\
0 & 0 & 0 & 1
\end{array}\right] .
\end{aligned}
$$

$\mathbf{T}_{j}^{A}$ and $\mathbf{T}_{j}^{N}$ represent the actual and nominal transformation matrix between $(j-1)$ th and $j$ th coordinate system, respectively. The deviation between them is expressed as

$$
\begin{aligned}
\mathbf{d T}_{j} & =\mathbf{T}_{j}^{A}-\mathbf{T}_{j}^{N} \\
& =\frac{\partial \mathbf{T}_{j}}{\partial \alpha_{j-1}} \Delta \alpha_{j-1}+\frac{\partial \mathbf{T}_{j}}{\partial a_{j-1}} \Delta a_{j-1}+\frac{\partial \mathbf{T}_{j}}{\partial d_{j}} \Delta d_{j}+\frac{\partial \mathbf{T}_{j}}{\partial \theta_{j}} \Delta \theta_{j} .
\end{aligned}
$$

Due to $\mathbf{d T}_{j}=\mathbf{T}_{j}^{N} \cdot \delta \mathbf{T}_{j}$ (plug (5) into (6)), it can be obtained that 


$$
\delta \mathbf{T}_{j}=\left[\begin{array}{cccc}
0 & -\cos \alpha_{j-1} \Delta \theta_{j} & \sin \alpha_{j-1} \Delta \theta_{j} & \Delta a_{j-1} \\
\cos \alpha_{j-1} \Delta \theta_{j} & 0 & -\Delta \alpha_{j-1} & a_{j-1} \cos \alpha_{j-1} \Delta \theta_{j}+\sin \alpha_{j-1} \Delta d_{j} \\
-\sin \alpha_{j-1} \Delta \theta_{j} & \Delta \alpha_{j-1} & 0 & -a_{j-1} \sin \alpha_{j-1} \Delta \theta_{j}+\cos \alpha_{j-1} \Delta d_{j} \\
0 & 0 & 0 & 0
\end{array}\right]
$$

Combining $\delta \mathbf{T}_{j}=\left[\begin{array}{cccc}0 & -\delta_{j z} & \delta_{j y} & d_{j x} \\ \delta_{j z} & 0 & -\delta_{j x} & d_{j y} \\ -\delta_{j y} & \delta_{j x} & 0 & d_{j z} \\ 0 & 0 & 0 & 0\end{array}\right]$ with (7), the following equation is obtained:

$$
\left[\begin{array}{l}
\mathbf{d}_{j} \\
\boldsymbol{\delta}_{j}
\end{array}\right]=\left[\begin{array}{cccc}
0 & \mathbf{k}_{j}^{1} & \mathbf{k}_{j}^{2} & \mathbf{k}_{j}^{3} \\
\mathbf{k}_{j}^{4} & 0 & 0 & \mathbf{k}_{j}^{5}
\end{array}\right]\left[\begin{array}{c}
\Delta \alpha_{j-1} \\
\Delta a_{j-1} \\
\Delta d_{j} \\
\Delta \theta_{j}
\end{array}\right]
$$

wherein $\mathbf{d}_{j}=\left[\begin{array}{lll}d_{j x} & d_{j y} & d_{j z}\end{array}\right]^{\mathrm{T}}$ and $\boldsymbol{\delta}_{j}=\left[\begin{array}{lll}\delta_{j x} & \delta_{j y} & \delta_{j z}\end{array}\right]^{\mathrm{T}}$ are the position deviation and orientation deviation of $j$ th coordinate system relative to $(j-1)$ th coordinate system, respectively. Meanwhile, $\mathbf{k}_{j}^{l}(l=1, \ldots, 5) \in \mathbb{R}^{3 \times 1}$. Consider

$$
\begin{aligned}
& \mathbf{k}_{j}^{1}=\left[\begin{array}{lll}
1 & 0 & 0
\end{array}\right]^{\mathrm{T}} ; \quad \mathbf{k}_{j}^{2}=\left[\begin{array}{lll}
0 & \sin \alpha_{i-1} & \cos \alpha_{i-1}
\end{array}\right]^{\mathrm{T}} ; \\
& \mathbf{k}_{j}^{3}=\left[\begin{array}{lll}
0 & a_{i-1} \cos \alpha_{i-1} & -a_{i-1} \sin \alpha_{i-1}
\end{array}\right]^{\mathrm{T}} ; \\
& \mathbf{k}_{j}^{4}=\left[\begin{array}{lll}
1 & 0 & 0
\end{array}\right]^{\mathrm{T}} ; \quad \mathbf{k}_{j}^{5}=\left[\begin{array}{lll}
0 & \sin \alpha_{i-1} & \cos \alpha_{i-1}
\end{array}\right]^{\mathrm{T}} .
\end{aligned}
$$

For the entire manipulator, the nominal transformation matrix from the coordinate system of the end-effector to the base coordinate system can be expressed as

$$
\mathbf{T}^{N}=\mathbf{T}_{1} \cdots \mathbf{T}_{j} \cdots \mathbf{T}_{n} .
$$

$\mathbf{T}^{A}$ and $\mathbf{T}^{N}$ represent the actual and nominal transformation matrix from the coordinate of the end-effector to the base coordinate system, respectively. Then,

$$
\begin{aligned}
\mathbf{T}^{A} & =\mathbf{T}^{N}+\mathbf{d} \mathbf{T}=\prod_{j=1}^{n}\left(\mathbf{T}_{j}^{N}+d \mathbf{T}_{j}\right) \\
& \approx \mathbf{T}^{N}+\sum_{j=1}^{n}\left(\mathbf{T}_{1} \cdots \mathbf{T}_{j-1} \cdot d \mathbf{T}_{j} \cdot \mathbf{T}_{j+1} \cdots \mathbf{T}_{n}\right),
\end{aligned}
$$

wherein $\mathbf{d T}$ is the differential of the transformation matrix from the coordinate of the end-effector to the base coordinate system. First-order approximation is used for (11). Then, according to $d \mathbf{T}_{j}=\mathbf{T}_{j} \cdot \delta \mathbf{T}_{j}$,

$$
\mathbf{d} \mathbf{T}=\sum_{j=1}^{n}\left(\mathbf{T}^{N} \cdot\left(\mathbf{T}_{j+1} \cdots \mathbf{T}_{n}\right)^{-1} \cdot \delta \mathbf{T}_{j} \cdot \mathbf{T}_{j+1} \cdots \mathbf{T}_{n}\right) .
$$

Define $\mathbf{U}_{i}=\mathbf{T}_{i} \cdot \mathbf{T}_{i+1} \cdots \mathbf{T}_{n}=\left[\begin{array}{cc}\mathbf{R}_{i}^{u} & \mathbf{p}_{i}^{u} \\ \mathbf{3}_{3 \times 3} & 1\end{array}\right]$. Combining with $\mathbf{d T}=\mathbf{T}^{N} \cdot \delta \mathbf{T}$, it is concluded as

$$
\begin{aligned}
\delta \mathbf{T} & =\sum_{j=1}^{n}\left(\mathbf{U}_{j+1}{ }^{-1} \cdot \delta \mathbf{T}_{j} \cdot \mathbf{U}_{j+1}\right) \\
& =\left[\begin{array}{cccc}
0 & -\delta_{z} & \delta_{y} & d_{x} \\
\delta_{z} & 0 & -\delta_{x} & d_{y} \\
-\delta_{y} & \delta_{x} & 0 & d_{z} \\
0 & 0 & 0 & 0
\end{array}\right] .
\end{aligned}
$$

$\mathbf{d}=\left[\begin{array}{lll}d_{x} & d_{y} & d_{z}\end{array}\right]^{\mathrm{T}}$ and $\boldsymbol{\delta}=\left[\begin{array}{lll}\delta_{x} & \delta_{y} & \delta_{z}\end{array}\right]^{\mathrm{T}}$ represent the position and orientation deviation; they can be expressed as

$$
\left[\begin{array}{l}
\mathbf{d} \\
\boldsymbol{\delta}
\end{array}\right]=\sum_{j=1}^{n}\left(\left[\begin{array}{cc}
\left(\mathbf{p}_{j+1}^{u} \times \mathbf{R}_{j+1}^{u}\right)^{\mathrm{T}} & \left(\mathbf{R}_{j+1}^{u}\right)^{\mathrm{T}} \\
\left(\mathbf{R}_{j+1}^{u}\right)^{\mathrm{T}} & \mathbf{0}_{3 \times 3}
\end{array}\right] \cdot\left[\begin{array}{l}
\boldsymbol{\delta}_{j} \\
\mathbf{d}_{j}
\end{array}\right]\right) .
$$

Combine (8) with (14); the following relationship is concluded:

$$
\left[\begin{array}{l}
\mathbf{d} \\
\boldsymbol{\delta}
\end{array}\right]=\left[\begin{array}{cccc}
\mathbf{M}_{1} & \mathbf{M}_{2} & \mathbf{M}_{3} & \mathbf{M}_{4} \\
\mathbf{M}_{5} & \mathbf{0}_{3 \times n} & \mathbf{0}_{3 \times n} & \mathbf{M}_{6}
\end{array}\right] \cdot\left[\begin{array}{c}
\Delta \boldsymbol{\alpha} \\
\Delta \mathbf{a} \\
\Delta \mathbf{d} \\
\Delta \boldsymbol{\theta}
\end{array}\right]
$$

wherein $\mathbf{M}_{l}(l=1, \ldots, 6) \in \mathbb{R}^{3 \times n}$ and $\Delta \boldsymbol{\alpha}, \Delta \mathbf{a}, \Delta \mathbf{d}, \Delta \boldsymbol{\theta} \in \mathbb{R}^{n \times 1}$, which can be expressed in detail as

$$
\begin{aligned}
& \Delta \boldsymbol{\alpha}=\left[\begin{array}{lll}
\Delta \alpha_{0} & \cdots & \Delta \alpha_{n-1}
\end{array}\right]^{\mathrm{T}} ; \quad \Delta \mathbf{a}=\left[\begin{array}{lll}
\Delta a_{0} & \cdots & \Delta a_{n-1}
\end{array}\right]^{\mathrm{T}} ; \\
& \Delta \mathbf{d}=\left[\begin{array}{lll}
\Delta d_{1} & \cdots & \Delta d_{n}
\end{array}\right]^{\mathrm{T}} ; \quad \Delta \boldsymbol{\theta}=\left[\begin{array}{lll}
\Delta \theta_{1} & \cdots & \Delta \theta_{n}
\end{array}\right]^{\mathrm{T}} ; \\
& \mathbf{M}_{1}^{j}=\left(\mathbf{p}_{j+1}^{u} \times \mathbf{R}_{j+1}^{u}\right)^{\mathrm{T}} \cdot \mathbf{k}_{j}^{4} ; \quad \mathbf{M}_{2}^{j}=\left(\mathbf{R}_{j+1}^{u}\right)^{\mathrm{T}} \cdot \mathbf{k}_{j}^{1} ; \\
& \mathbf{M}_{3}^{j}=\left(\mathbf{R}_{j+1}^{u}\right)^{\mathbf{T}} \cdot \mathbf{k}_{j}^{2} \text {; } \\
& \mathbf{M}_{4}^{j}=\left(\mathbf{p}_{j+1}^{u} \times \mathbf{R}_{j+1}^{u}\right)^{\mathbf{T}} \cdot \mathbf{k}_{j}^{5}+\left(\mathbf{R}_{j+1}^{u}\right)^{\mathbf{T}} \cdot \mathbf{k}_{j}^{3} ; \\
& \mathbf{M}_{5}^{j}=\left(\mathbf{R}_{j+1}^{u}\right)^{\mathbf{T}} \cdot \mathbf{k}_{j}^{4} ; \quad \mathbf{M}_{6}^{j}=\left(\mathbf{R}_{j+1}^{u}\right)^{\mathbf{T}} \cdot \mathbf{k}_{j}^{5} ; \quad j=0, \ldots, n .
\end{aligned}
$$

Then the position deviation of the end-effector can be obtained from (15):

$$
X(\Theta)=\left\|\mathbf{M}_{1} \Delta \boldsymbol{\alpha}+\mathbf{M}_{2} \Delta \mathbf{a}+\mathbf{M}_{3} \Delta \mathbf{d}+\mathbf{M}_{4} \Delta \boldsymbol{\theta}\right\| .
$$

So far, relationship between deviations of kinematics parameters and position error is derived. Meanwhile, 


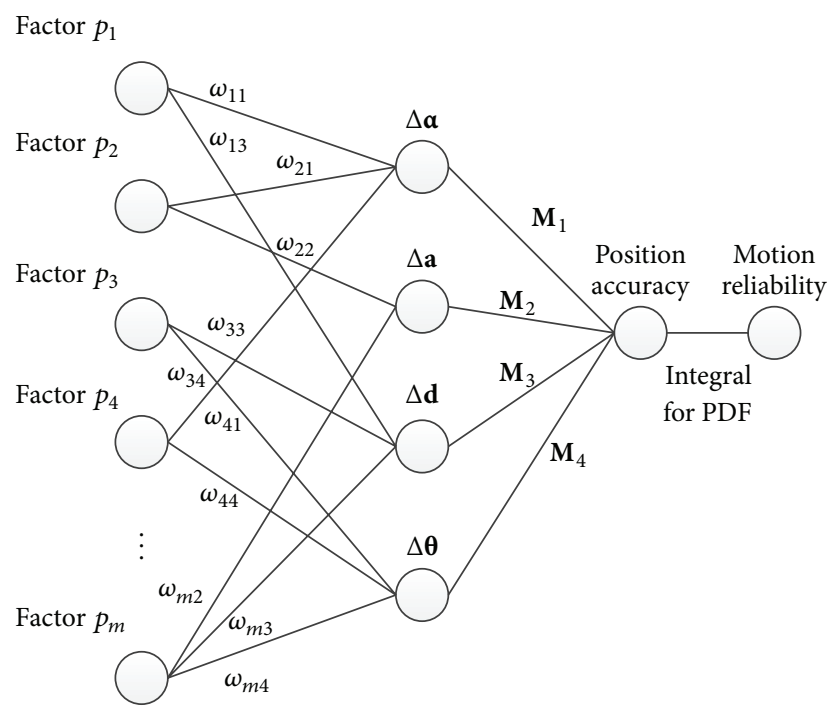

FIGURE 2: Influence relationships between factors and motion reliability.

he contribution of each parameter to the position accuracy is also obtained as matrix $\mathbf{M}$. In fact, the deviations of kinematics parameters are the intermediate variables to reflect the influence of factors related to position accuracy. Then motion reliability can be obtained via the integral for the PDF of position accuracy. The relationships are shown in Figure 2.

Figure 2 gives a schematic view of the influence relationship between factors and motion reliability. When different factors are considered, their effect can be delivered via kinematics parameters. Thus, each kinematics parameters can be expressed as a function, such as

$$
\Delta \boldsymbol{\theta}=f\left(p_{i}, \ldots, p_{j}\right), \quad 1 \leq i<j \leq m,
$$

wherein $p_{i}$ is the $i$ th factor which can deliver the influence on the position accuracy to parameter $\boldsymbol{\theta}$. Other kinematics parameters can be expressed in a similar form. If (18) can be linearized, the contribution weight of each factor can be obtained as $\omega$ which is listed in Figure 2. However, many problems exist in the derivation and linearization of functions like (18), which will not be discussed further in the paper.

2.3. Motion Reliability Models of Path Planning Task. In summary, combining (17) with (2) and (4), the motion reliability models for point-to-point path planning and continuous trajectory tracking can be obtained, respectively. The motion reliability model for point-point path planning is expressed as

$$
\begin{aligned}
R & =\operatorname{Pr}\left\{\left\|\mathbf{M}_{1} \Delta \boldsymbol{\alpha}+\mathbf{M}_{2} \Delta \mathbf{a}+\mathbf{M}_{3} \Delta \mathbf{d}+\mathbf{M}_{4} \Delta \boldsymbol{\theta}\right\|<l_{p}\right\} \\
& =\int_{0}^{l_{p}} p(x) d x .
\end{aligned}
$$

The motion reliability model for continuous trajectory tracking is expressed as

$R$

$$
\begin{aligned}
& =\operatorname{Pr}\left\{\bigcap_{i=1}^{m \rightarrow \infty}\left(\left\|\mathbf{M}_{1} \Delta \boldsymbol{\alpha}+\mathbf{M}_{2} \Delta \mathbf{a}+\mathbf{M}_{3} \Delta \mathbf{d}+\mathbf{M}_{4} \Delta \boldsymbol{\theta}\right\|_{i}<l_{c}\right)\right\} \\
& =\int_{0}^{l_{c}}\left(\ldots\left(\int_{0}^{l_{c}}\left(\int_{0}^{l_{c}} p\left(x_{1}, x_{2}, \ldots, x_{m}\right) d x_{1}\right) d x_{2}\right) \ldots\right) d x_{m} .
\end{aligned}
$$

So far, models of motion reliability for path planning task are obtained. According to (19) and (20), the models mean to obtain the probability that the position deviation guarantees the position accuracy threshold. Meanwhile, deviations of kinematics parameters are taken into account, which successfully introduces the influence of factors related to position accuracy into the models. Then the models should be calculated to achieve motion reliability evaluation, which will be discussed in the next section.

\section{Simplification and Calculation for Motion Reliability Model}

In order to calculate motion reliability models of path planning task, samples of position deviations should be firstly obtained by introducing errors into factors. As a basis, PDF related to the samples can be computed and the motion reliability can be calculated by integral for PDF. For point-to-point path planning, motion reliability can be calculated according to (19) by one dimensional integral for PDF. However, it is more complicated to calculate the motion reliability of continuous trajectory tracking because there are so many trajectory points. The PDF in the task is concerned with position deviations of entire trajectory points; multidimensional integrals for PDF should be carried out in motion reliability evaluation, which brings about incredible computation cost simultaneously. In order to solve model (20) simply, approach of equivalent extreme value is introduced to simplify the model.

3.1. Simplification for Motion Reliability Model. Define theorem as follows.

Theorem 1. Suppose $X_{1}, X_{2}, \ldots, X_{m}$ are $m$ random variables; define the maximum value of $X_{1}, X_{2}, \ldots, X_{m}$ as $W_{\max }=$ $\max _{1 \leq i \leq m}\left(X_{i}\right)$; then it goes that $\operatorname{Pr}\left\{\bigcap_{i=1}^{m}\left(X_{i}<a\right)\right\}=\operatorname{Pr}\left\{W_{\max }<\right.$ a\}.

Proof. Firstly, prove $\operatorname{Pr}\left\{\left(X_{1}<a\right) \bigcap\left(X_{2}<a\right)\right\}=\operatorname{Pr}\left\{W_{\max }^{(2)}<\right.$ a\}.

Define $p\left(x_{1}, x_{2}\right)$ as the PDF related to $X_{1}, X_{2}$; then,

$$
\begin{aligned}
\operatorname{Pr} & \left\{\left(X_{1}<a\right) \bigcap\left(X_{2}<a\right)\right\} \\
& =\iint_{x_{1}<a, x_{2}<a} p\left(x_{1}, x_{2}\right) d x_{1} d x_{2} \\
& =\int_{-\infty}^{a}\left(\int_{-\infty}^{a} p\left(x_{1}, x_{2}\right) d x_{1}\right) d x_{2} .
\end{aligned}
$$


Because the maximum value of $X_{1}, X_{2}$ can be expressed as

$$
W_{\max }^{(2)}= \begin{cases}X_{1} & \text { if } X_{1} \geq X_{2} \\ X_{2} & \text { else }\end{cases}
$$

it can be derived that

$$
\begin{aligned}
\operatorname{Pr}\left\{W_{\max }^{(2)}<a\right\}= & \operatorname{Pr}\left\{\max \left(X_{1}, X_{2}\right)<a\right\} \\
= & \iint_{x_{2}<x_{1}, x_{1}<a} p\left(x_{1}, x_{2}\right) d x_{1} d x_{2} \\
& +\iint_{x_{1}<x_{2}, x_{2}<a} p\left(x_{1}, x_{2}\right) d x_{1} d x_{2} \\
= & \int_{-\infty}^{a}\left(\int_{x_{2}}^{a} p\left(x_{1}, x_{2}\right) d x_{1}\right) d x_{2} \\
& +\int_{-\infty}^{a}\left(\int_{-\infty}^{x_{2}} p\left(x_{1}, x_{2}\right) d x_{1}\right) d x_{2} \\
= & \int_{-\infty}^{a}\left(\int_{-\infty}^{a} p\left(x_{1}, x_{2}\right) d x_{1}\right) d x_{2} .
\end{aligned}
$$

Consider $\operatorname{Pr}\left\{\left(X_{1}<a\right) \bigcap\left(X_{2}<a\right)\right\}=\operatorname{Pr}\left\{W_{\max }^{(2)}<a\right\}$ is proved.

The maximum value of $X_{1}, X_{2}, \ldots, X_{i}$ is expressed as $W_{\max }^{(i)}=\max \left(X_{1}, X_{2}, \ldots, X_{i}\right)$. Define $W_{\max }^{(1)}=X_{1}, W_{\max }=$ $W_{\max }^{(m)}$, and then $W_{\max }^{(i)}=\max \left(W_{\max }^{(i-1)}, X_{i}\right)$.

Combing with $\operatorname{Pr}\left\{\left(X_{1}<a\right) \bigcap\left(X_{2}<a\right)\right\}=\operatorname{Pr}\left\{W_{\max }^{(2)}<a\right\}$, it goes that

$$
\begin{aligned}
\operatorname{Pr} & \left\{\bigcap_{i=1}^{m}\left(X_{i}<a\right)\right\} \\
& =\operatorname{Pr}\left\{\left\{\left(X_{1}<a\right) \cap\left(X_{2}<a\right)\right\} \bigcap_{i=3}^{m}\left(X_{i}<a\right)\right\} \\
& =\operatorname{Pr}\left\{\left\{\left(W_{\max }^{(2)}<a\right) \cap\left(X_{3}<a\right)\right\} \bigcap_{i=4}^{m}\left(X_{i}<a\right)\right\} \\
& =\cdots=\operatorname{Pr}\left\{\left(W_{\max }^{(m-1)}<a\right) \cap\left(X_{m}<a\right)\right\} \\
& =\operatorname{Pr}\left\{W_{\max }^{m}<a\right\}=\operatorname{Pr}\left\{W_{\max }<a\right\} .
\end{aligned}
$$

According to the theorem, define $W_{\max }(\Theta)=$ $\max _{1 \leq i \leq m}\left(X_{i}(\Theta)\right)$ as the maximum position deviation of entire trajectory points; then motion reliability model of (20) can be turned into

$$
\begin{aligned}
R & =\operatorname{Pr}\left\{\bigcap_{i=1}^{m \rightarrow \infty}\left(X_{i}(\Theta)<l\right)\right\}=\operatorname{Pr}\left\{W_{\max }(\Theta)<l\right\} \\
& =\int_{0}^{\infty} p(w) d w,
\end{aligned}
$$

wherein $p(w)$ represents the PDF related to the maximum position deviation of the manipulator. Thus, the multidimensional integrals for the PDF related to position deviation of the entire trajectory points can be turned into one dimensional integral for PDF related to the maximum position deviation. This method is called the approach of equivalent extreme value, with which the motion reliability model of continuous trajectory tracking can be simplified and easily solved.

3.2. Calculation for PDF Based on Maximum Entropy Principle. After the model is simplified, the core of motion reliability evaluation is the calculation of PDF. Generally, Monte Carlo method can be used to solve any problems about probability distribution. However, massive samples are needed and computation cost is high. In order to achieve fast calculation of PDF with high precision, the information entropy is introduced and a method based on the principle of maximum entropy is proposed. The principle of maximum entropy [27] was firstly proposed by Jaynes, which aimed at choosing the probability distribution at the maximum state of information entropy. The distribution obtained at maximum entropy state is considered to be the most realistic. Meanwhile, the probability distribution based on the maximum entropy principle has high computation precision, which has been verified in [15] by comparison with Monte Carlo method. Therefore, the method based on the maximum entropy principle has huge advantages in fast and precise computation.

When evaluating the motion reliability of continuous trajectory tracking, the PDF can be calculated as follows. Suppose $w$ as the maximum position deviation of the entire trajectory, and $p(w)$ represents the PDF of $w$. Then the information entropy of $w$ can be expressed as

$$
H(w)=-\int p(w) \ln p(w) d w
$$

Equation (26) shows the relationship between the entropy and PDF. The PDF of continuous trajectory tracking task should meet the constraints shown in (27a) and (27b), wherein constraint (27a) means that the integral for PDF equals 1 . Constraint (27b) is a group of constraints, which denote the $\gamma_{i}$ moment of $w$. The number of constraints (27b) depends on the moment $s$. During constraint (27b), the moment $\gamma_{i}$ can be either integer or fraction:

$$
\begin{gathered}
\int_{0}^{\infty} p(w) d w=1 \\
\int_{0}^{\infty} w^{\gamma_{i}} p(w) d w=u_{i}, \quad i=1,2, \ldots, s .
\end{gathered}
$$


As the maximum entropy principle describes, the PDF is the most realistic when the information entropy is maximum. In order to find the maximum value of entropy, Lagrange multiplier method [28] is introduced. Suppose $1,\left(\lambda_{0}-\right.$ $1), \lambda_{1}, \ldots, \lambda_{s}$ as Lagrange multipliers; the Lagrange function related to information entropy is established as

$$
\begin{aligned}
L= & \int_{0}^{\infty} p(w) \ln p(w) d w+\left(\lambda_{0}-1\right)\left[\int_{0}^{\infty} p(w) d w-1\right] \\
& +\sum_{i=1}^{s} \lambda_{i}\left[\int_{0}^{\infty} w^{\gamma_{i}} p(w) d w-u_{i}\right]
\end{aligned}
$$

Take partial derivative with respect to $p(w)$, and make $\partial L / \partial p(w)=0$. Then,

$$
\frac{\partial L}{\partial p(w)}=\int_{0}^{\infty}\left[\ln p(w)+\lambda_{0}+\sum_{i=1}^{s} \lambda_{i} w^{\gamma_{i}}\right] d w=0 .
$$

The expression of $p(w)$ at the maximum entropy state can be derived:

$$
\widehat{p}(w)=\exp \left(-\lambda_{0}-\sum_{i=1}^{s} \lambda_{i} w^{\gamma_{i}}\right)
$$
(30):

Meanwhile, $\lambda_{0}$ can be obtained by plugging (27a) into

$$
\lambda_{0}=\ln \left[\int_{0}^{\infty} \exp \left(-\sum_{i=1}^{s} \lambda_{i} w^{\gamma_{i}}\right) d w\right] .
$$

In order to calculate the unknown constants $\lambda_{i}, \gamma_{i}(i=$ $1, \ldots s)$ in $\widehat{p}(w)$, Kullback-Leibler (K-L) divergence [9] is introduced, which represents the deviation between $\widehat{p}(w)$ and the actual value. The expression is shown as follows:

$$
K[p, \widehat{p}]=\int p(w) \ln \left[\frac{p(w)}{\widehat{p}(w)}\right] d w
$$

Substitute (26), (27a), (27b), (30), and (31) into (31):

$$
\begin{aligned}
K[p, \widehat{p}]= & -H(w)+\ln \left[\int_{0}^{\infty} \exp \left(-\sum_{i=1}^{s} \lambda_{i} w^{\gamma_{i}}\right) d w\right] \\
& +\sum_{i=1}^{s} \lambda_{i} u_{i} .
\end{aligned}
$$

When $\widehat{p}(w)$ is the closest to the actual value, the K-L divergence is minimal. Suppose $\lambda=\left(\lambda_{1}, \ldots, \lambda_{s}\right)$ and $\gamma=$ $\left(\gamma_{1}, \ldots, \gamma_{s}\right)$. Since $H(w)$ is unrelated to $\lambda$ and $\gamma, K[p, \widehat{p}]$ is minimal, meaning the following formula is minimal:

$$
I(\boldsymbol{\lambda}, \boldsymbol{\gamma})=\ln \left[\int_{0}^{\infty} \exp \left(-\sum_{i=1}^{s} \lambda_{i} w^{\gamma_{i}}\right) d w\right]+\sum_{i=1}^{s} \lambda_{i} u_{i} .
$$

According to (27b), the $\gamma_{i}$ moment of $w$ equals $u_{i}$. $u_{i}$ can be approximately expressed as $u_{i}=(1 / N) \sum_{k=1}^{N} w^{\gamma_{i}}$ based on moment estimation, where $N$ is the number of samples. Therefore, the calculation of the $\operatorname{PDF} p(w)$ is turned into

$$
\begin{aligned}
& \text { Find: } \gamma_{i} \text { and } \lambda_{i} \quad(i=1, \ldots, s) \\
& \text { Min: } I(\boldsymbol{\lambda}, \boldsymbol{\gamma})=\ln \left[\int_{0}^{\infty} \exp \left(-\sum_{i=1}^{s} \lambda_{i} w^{\gamma_{i}}\right) d w\right] \\
& +\sum_{i=1}^{s}\left(\frac{\lambda_{i}}{N} \sum_{k=1}^{N} w^{\gamma_{i}}\right) .
\end{aligned}
$$

Set the initial value of $\gamma$; then $\gamma$ and $\lambda$ can be iteratively obtained via search algorithm until $I(\boldsymbol{\lambda}, \boldsymbol{\gamma})$ is minimum. Substituting $\gamma$ and $\lambda$ into (30) and (31), the value of $\lambda_{0}$ and the expression of $p(w)$ can be achieved. Finally, the motion reliability of continuous trajectory tracking can be calculated by one dimensional integral for $p(w)$.

3.3. Process for Calculating Motion Reliability Models of Path Planning Task. Based on the analysis above, the process for calculating the motion reliability of path planning task can be concluded, which is also shown in Figure 3.

(a) Motion reliability model is firstly established for a particular path planning task. For model of continuous trajectory tracking, the approach of equivalent extreme value is introduced. As a result, multidimensional integrals for PDF related to the entire trajectory points are turned into one dimensional integral for PDF related to the maximum position deviation.

(b) Introduce errors into factors related to position accuracy of the model, and simulate the path planning task for multiple times. For point-to-point path planning, position deviation at the target point of each simulation is obtained. For continuous trajectory tracking, position deviations at each trajectory point are obtained.

(c) For point-to-point path planning, the position deviation at the target point of each simulation is selected as samples. For continuous trajectory tracking, the maximum position deviation of each simulation is selected as samples.

(d) Lagrange function about information entropy of position deviation samples is established, and the expression of PDF related to position deviation samples is derived at the state of maximum entropy.

(e) The unknown coefficients of the PDF are calculated by using K-L divergence. Then the motion reliability evaluation is achieved by one dimensional integral for PDF.

\section{Simulation on Motion Reliability Evaluation of Path Planning Task}

A 7-DOF manipulator is used for motion reliability evaluation of path planning task. The coordinate systems are defined as shown in Figure 4, while the nominal DH parameters are 


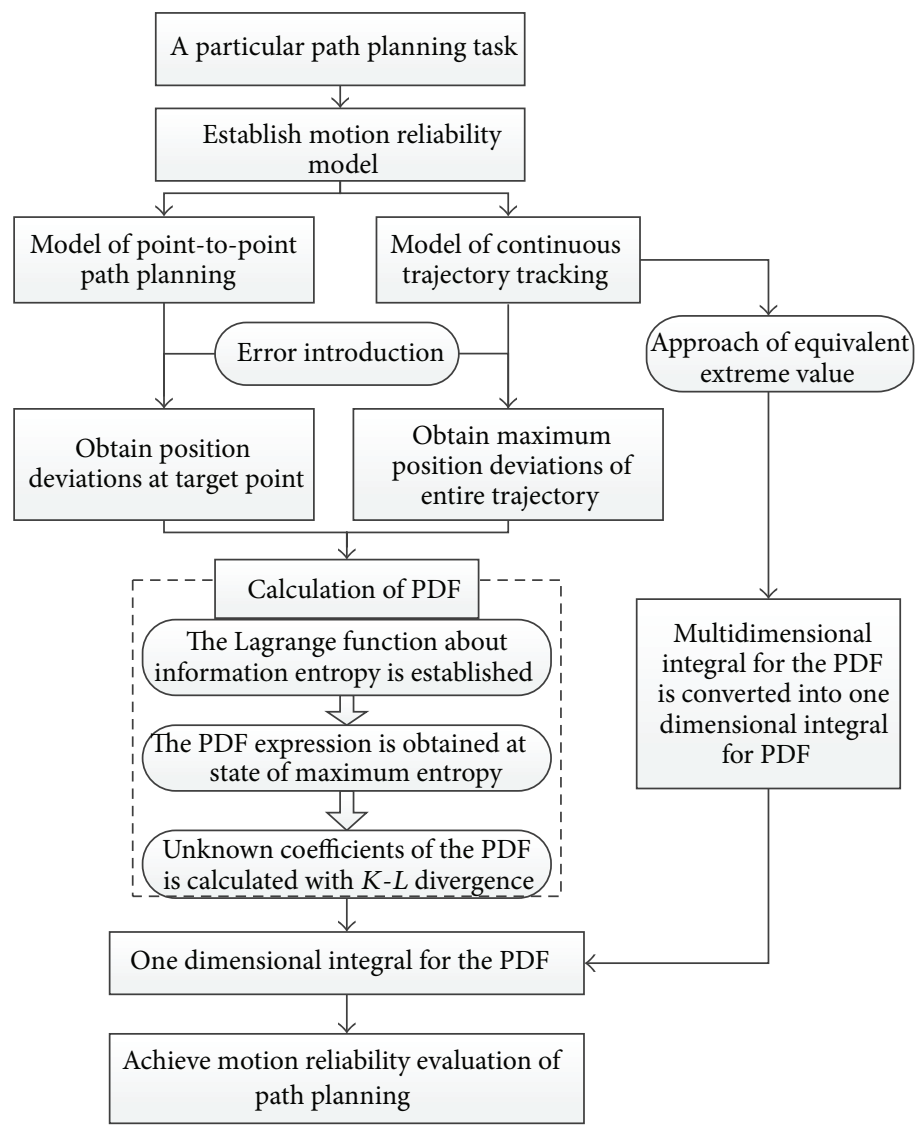

FIGURE 3: Process for calculating the motion reliability model of path planning task.

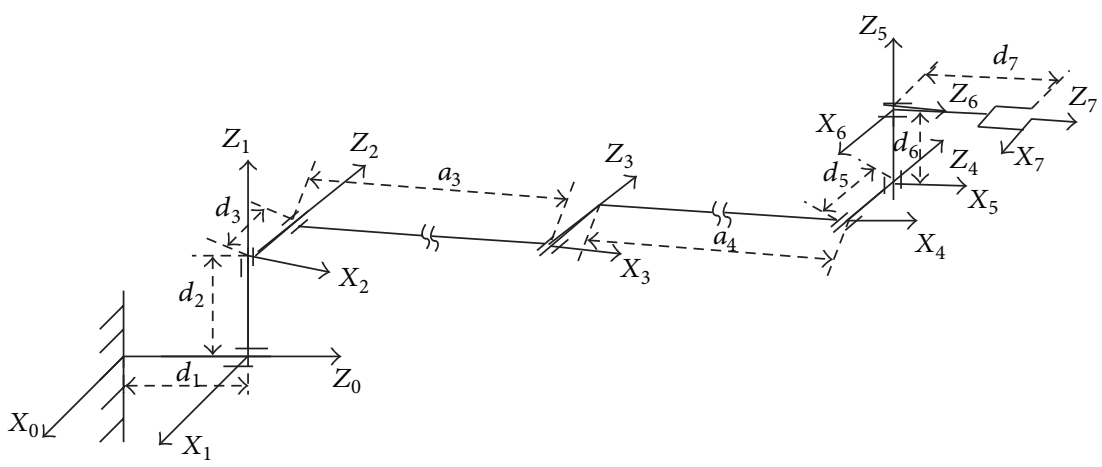

FIGURE 4: The coordinate systems of 7-DOF manipulator.

listed in Table 1. A particular continuous trajectory task is defined for simulation as follows.

The initial configuration of manipulator is set as

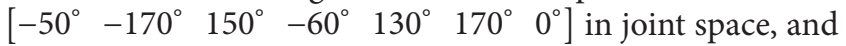
the position of the end-effector at this configuration is denoted by point $A$. The target pose is set as $\left[\begin{array}{llllll}9.6 & 0 & 3 & -1 & -0.5 & -2\end{array}\right]$, which is denoted by point $B$. Continuous trajectory tracking task is carried out from $A$ to $B$.
Task cycle is $20 \mathrm{~s}$, and control cycle is $0.05 \mathrm{~s}$. As a result, 400 trajectory points are planned during the task.

\subsection{Motion Reliability Evaluation of a Particular Path Planning} Task. During the motion reliability evaluation of task, errors are introduced into kinematics parameters. Errors following normal distribution $N\left(0,0.001^{2}\right)$ (unit: $\mathrm{m}$ ) are introduced into a, d, and errors following normal distribution 
TABLE 1: The nominal DH parameters of 7-DOF manipulator.

\begin{tabular}{lcccc}
\hline Link $i$ & $\theta_{i}\left(^{\circ}\right)$ & $d_{i}(\mathrm{~m})$ & $a_{i-1}(\mathrm{~m})$ & $\alpha_{i-1}\left({ }^{\circ}\right)$ \\
\hline 1 & 0 & 0.6 & 0 & 90 \\
2 & 90 & 0.5 & 0 & -90 \\
3 & 0 & 0.5 & 5 & 0 \\
4 & 0 & 0.5 & 5 & 0 \\
5 & 0 & 0.5 & 0 & 90 \\
6 & -90 & 0.5 & 0 & -90 \\
7 & 0 & 0.6 & 0 & 0 \\
\hline
\end{tabular}

$N\left(0,0.01^{2}\right)$ (unit: ${ }^{\circ}$ ) are introduced into $\boldsymbol{\alpha}, \boldsymbol{\theta}$. The task is simulated for 500 times and position deviations of the entire trajectory points are collected for each simulation $\left\{X_{i}(\Theta)\right\}^{500}(i=1, \ldots, 400)$. The maximum position deviation is selected from each simulation to obtain 500 samples $\left\{X_{\max }(\Theta)\right\}^{500}$.

With the samples of maximum position deviation, PDF of the continuous trajectory tracking task is calculated. The initial value of $\gamma$ is set as $\gamma=\left[\begin{array}{lll}0.5 & -1 & 0.5\end{array}\right]$, and the order of moment is set as $s=3$. Search algorithm is carried out according to (35). $\lambda, \gamma$ are obtained as $\left[\begin{array}{ll}\gamma & \lambda\end{array}\right]=$ $\left[\begin{array}{llllll}1.1888 & -0.2103 & -0.4454 & 7.8457 & 1.3470 & 4.3483\end{array}\right]$ and $\lambda_{0}=-11.5896$ when $I(\lambda, \gamma)$ is minimum. As a basis, the curve of PDF related to the position accuracy threshold is obtained as in Figure 5. Via one dimensional integral for the PDF, the motion reliability can be calculated at different accuracy threshold, which is shown in Figure 6.

Meanwhile, the PDF and probability curves calculated by Monte Carlo method are also provided in Figures 5 and 6. With Monte Carlo method, the planning is simulated for 10000 times to obtain results. By comparison, the PDF and probability curves calculated with maximum entropy principle have the same accuracy with the results obtained with Monte Carlo method. At the same accuracy threshold, the deviation of calculated probability with the two methods is only about $1 \%$. For example, when accuracy threshold is $l=0.008 \mathrm{~m}$, the motion reliability is 0.9167 calculated by maximum entropy principle, while it equals 0.9064 with Monte Carlo method. It is verified that the evaluation result with maximum entropy principle has high precision.

Besides, huge computation cost and samples requirement limit Monte Carlo method in computing the PDF of trajectory tracking task. Nearly 3000 seconds are used to handle the 10000 samples to achieve the PDF. However, the method based on the maximum entropy principle needs fewer samples (just 500 samples), which greatly reduces the computation cost. In conclusion, the motion reliability evaluation method based on the maximum entropy principle is feasible with high efficiency and computation precision.

4.2. Motion Reliability Evaluation with Different Trajectory Points. When evaluating motion reliability of continuous tracking task, the number of trajectory points will have influence on the motion reliability. If the number is small, the characteristics of the trajectory cannot be reflected completely, which leads to a credible evaluation result. In

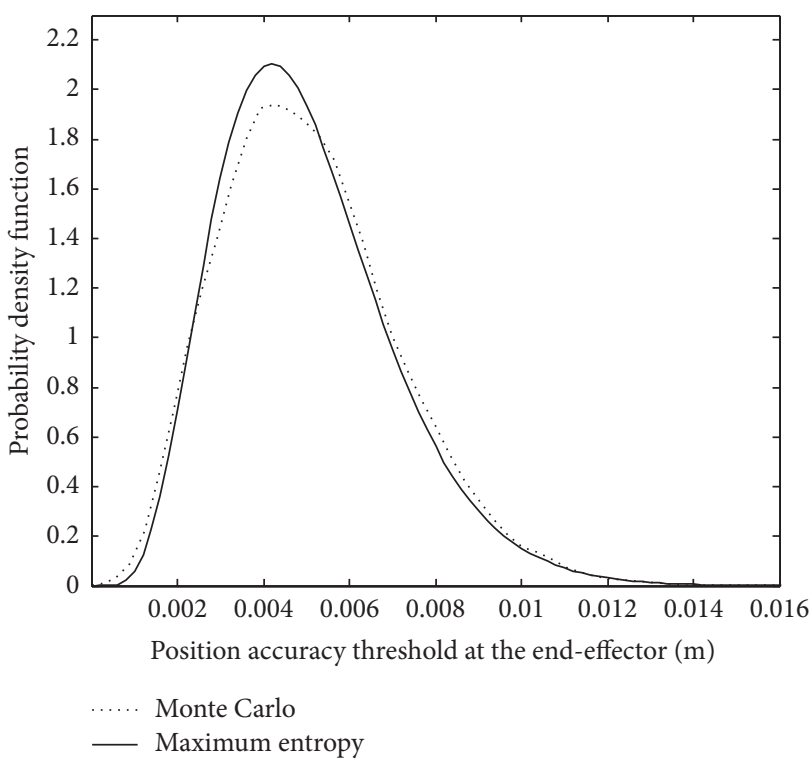

Figure 5: The PDF related to position accuracy threshold.

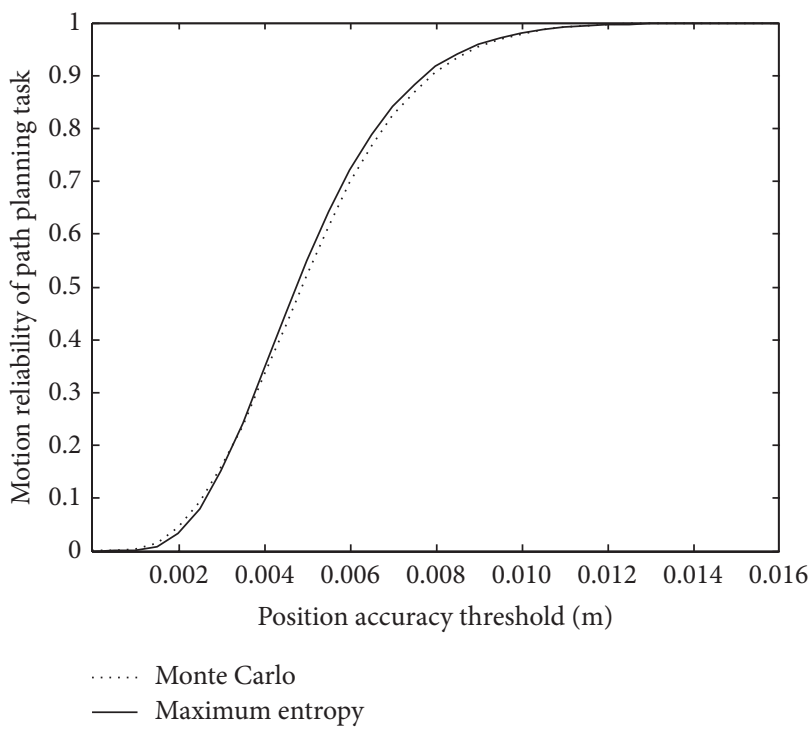

FIGURE 6: The motion reliability varies with accuracy requirement.

addition, with the number increase, the computation cost will become higher. Therefore, proper number of trajectory points should be selected by taking both computation efficiency and precision into account.

The trajectory $A \rightarrow B$ mentioned above is taken as an example, and the motion reliability of the task is evaluated with different trajectory points. The position accuracy threshold is set as $0.007 \mathrm{~m}$. Motion reliability varies with the number of trajectory points, which is shown in Figure 7. It is obvious that when the number of trajectory points is small, the motion reliability changes greatly with the number of trajectory points, because small numbers of trajectory points cannot reflect the characteristics of the trajectory completely. After the number is larger than 200 , the reliability becomes stable. 


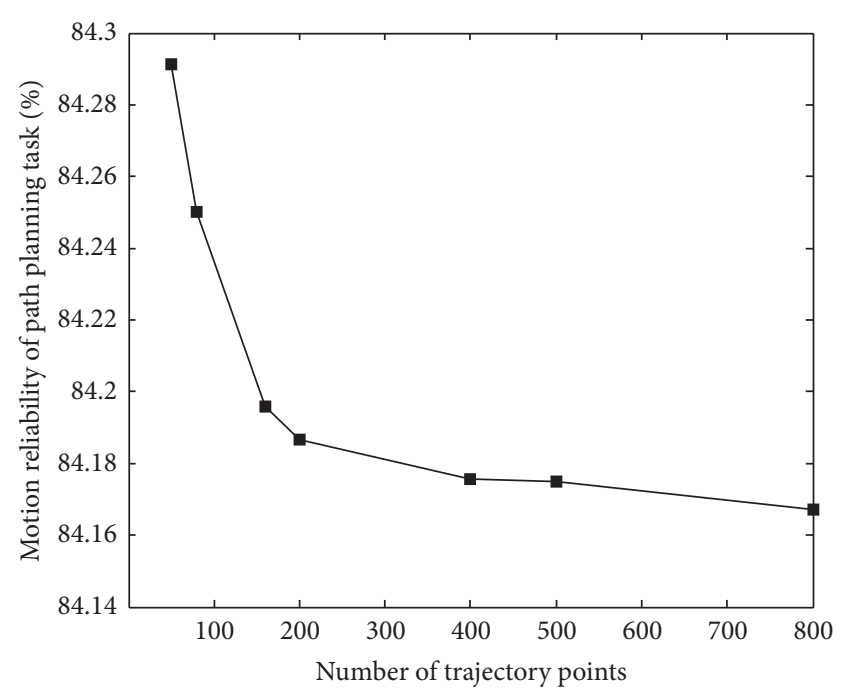

FIGURE 7: The motion reliability varies with trajectory points.

After the number is larger than 400, the reliability is nearly constant. The increased trajectory points only bring about higher computation cost in motion reliability evaluation. In summary, 400-500 is the proper number for the trajectory $A \rightarrow B$, which can completely reflect the characteristics of the trajectory and show good operational efficiency.

Besides, trajectory points are determined by task cycle and control cycle. The motion reliability evaluation will benefit from the proper number of trajectory points, which also indicates that the arrangement for task cycle and control cycle of the task is reasonable. In this way, reasonable arrangement for trajectory points is the basis to achieve fast and precise motion reliability evaluation.

\subsection{Contributions of Factors Related to Motion Reliability.} Factors influencing the position accuracy of manipulator can be included into the model of motion reliability. Via introducing errors into them, motion reliability of a particular path planning task can be evaluated, which is achieved in Section 4.1. In this section, the contributions of factors related to the motion reliability are discussed. $\boldsymbol{\alpha}, \boldsymbol{\theta}$ and $\mathbf{a}, \mathbf{d}$ are differed into two groups for different units. Errors following the same normal distribution are introduced into each group, respectively. $N\left(0,0.01^{2}\right)\left(^{\circ}\right)$ is introduced into $\boldsymbol{\alpha}, \boldsymbol{\theta}$, and the decrease in motion reliability caused by $\boldsymbol{\alpha}, \boldsymbol{\theta}$ is shown in Figure $8(\mathrm{a}) . N\left(0,0.001^{2}\right)(\mathrm{m})$ is introduced into $\mathbf{a}$, d; the decrease in motion reliability caused by $\mathbf{a}, \mathbf{d}$ is shown in Figure 8(b).

It is obvious that decreases caused in motion reliability are different within different position accuracy threshold for one factor. Compared with the matrix $\mathbf{M}$ in (17) which shows the contribution of factor at a moment in the task, Figure 8 can comprehensively reflect the influence of the factor on motion reliability during the entire task cycle. Further, the decrease in reliability caused by $\boldsymbol{\theta}$ is larger than $\boldsymbol{\alpha}$ within any accuracy threshold, while the decreases caused by a and $\mathbf{d}$ are basically the same. The decrease values in motion reliability caused by
TABLE 2: Decrease in motion reliability caused by single factor and combined effect.

\begin{tabular}{lccc}
\hline $\begin{array}{l}\text { Angular } \\
\text { factors }\end{array}$ & $\begin{array}{c}\text { Stand. deviation } \\
\text { of error }\left(^{\circ}\right)\end{array}$ & $\begin{array}{c}\text { Decrease of } \\
\text { reliability }\end{array}$ & Coupling effect \\
\hline $\boldsymbol{\alpha}$ & 0.01 & 0.08733 & 0.3809 \\
$\boldsymbol{\theta}$ & 0.01 & 0.1197 & Coupling effect \\
\hline $\begin{array}{l}\text { Length } \\
\text { factors }\end{array}$ & $\begin{array}{c}\text { Stand. deviation } \\
\text { of error }(\mathrm{m})\end{array}$ & $\begin{array}{c}\text { Decrease of } \\
\text { reliability }\end{array}$ & Con \\
\hline $\mathbf{a}$ & 0.001 & 0.1174 & 0.3608 \\
$\mathbf{d}$ & 0.001 & 0.1121 & \\
\hline
\end{tabular}

different factors at position accuracy threshold $0.004 \mathrm{~m}$ are also given out in the figure. Thus, the contributions can be expressed as $\boldsymbol{\theta}>\boldsymbol{\alpha}, \mathbf{a} \approx \mathbf{d}$ with the same errors. Meanwhile, the factors have coupling effect on motion reliability. The decreases in reliability caused by factors are listed at the accuracy threshold $0.004 \mathrm{~m}$ in Table 2.

In Table 2, when factors with the same unit act together, the decrease of reliability is greater than the sum of the decrement when they act separately. For example, decrement caused by $\boldsymbol{\alpha}$ and $\boldsymbol{\theta}$ is larger than the sum of decrement caused by them separately. Besides, when the standard deviations of introduced errors are different, the PDF and decrease in motion reliability are shown as in Figure 9 by analyzing factor $\boldsymbol{\alpha}$. It can be concluded that the decrease in motion reliability depends on the position accuracy threshold and standard deviations of errors, and the variation of which can be expressed by quadric surface as the figure shows. At the same position accuracy threshold, with the increase of standard deviation in errors, the decrease in motion reliability becomes larger. Variations of other factors can be expressed in similar ways, which are not given out here.

Thus the contributions of kinematics parameters to motion reliability are analyzed, and the coupling effect of parameters is discussed, too. Meanwhile, the variation according to the position accuracy threshold and standard deviation of errors is derived. As a result, the importance of each kinematics parameter in improving motion reliability can be obtained. When improving the motion reliability of manipulator, factors with high contribution should be given high priority to handle. According to the different contributions of parameters, the strategy to achieve motion reliability improvement is discussed in the next section.

\subsection{Discussion of Trajectory Optimization Based on Maximiz-} ing Motion Reliability. Since $\mathbf{a}, \mathbf{d}, \boldsymbol{\alpha}$, and $\boldsymbol{\theta}$ are easy to be observed and handled during task, the motion reliability can be improved by reducing the errors in these factors based on the contributions to motion reliability. For example, since $\boldsymbol{\theta}$ contributes more than $\mathbf{a}, \mathbf{d}$, and $\boldsymbol{\alpha}$, based on decreasing errors with kinematics calibration, the accuracy of $\boldsymbol{\theta}$ should be improved as much as possible. Thus, aiming at maximizing motion reliability, a trajectory optimization method is discussed via analyzing the contribution of $\boldsymbol{\theta}$.

Errors following normal distribution with different standard deviation are introduced into $\boldsymbol{\theta}$. Motion reliability 

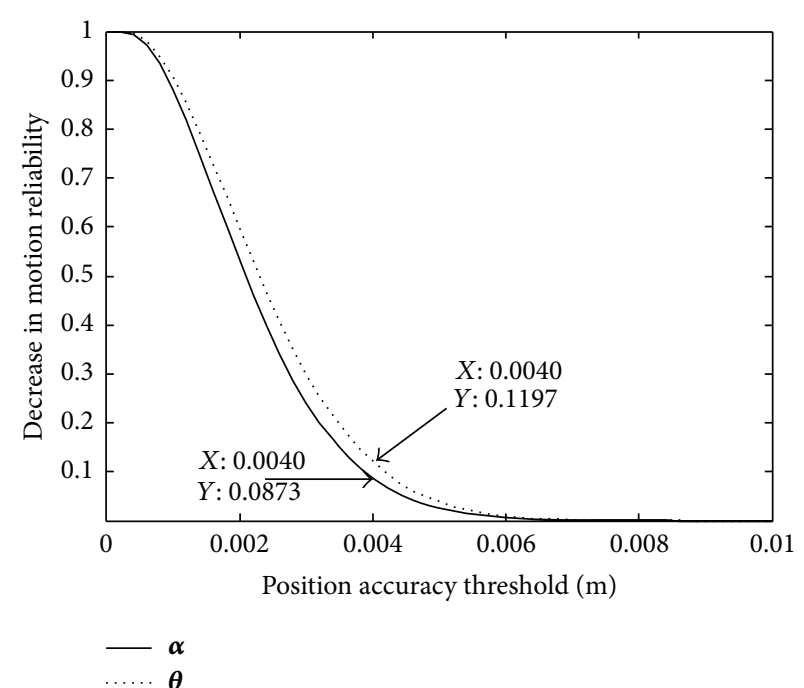

(a) Errors introduced into $\boldsymbol{\alpha}, \boldsymbol{\theta}$

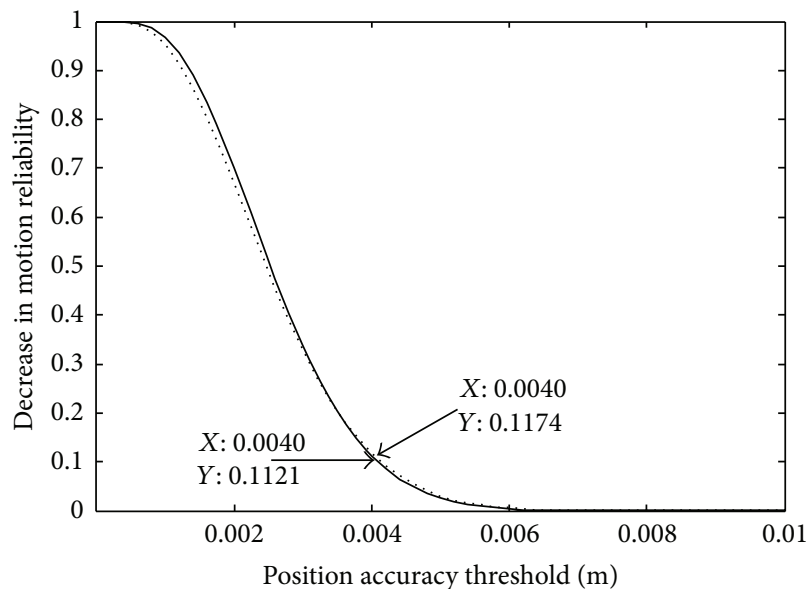

- a

(b) Errors introduced into a, $\mathbf{d}$

FIGURE 8: Decrease in motion reliability caused by factors with the same error.

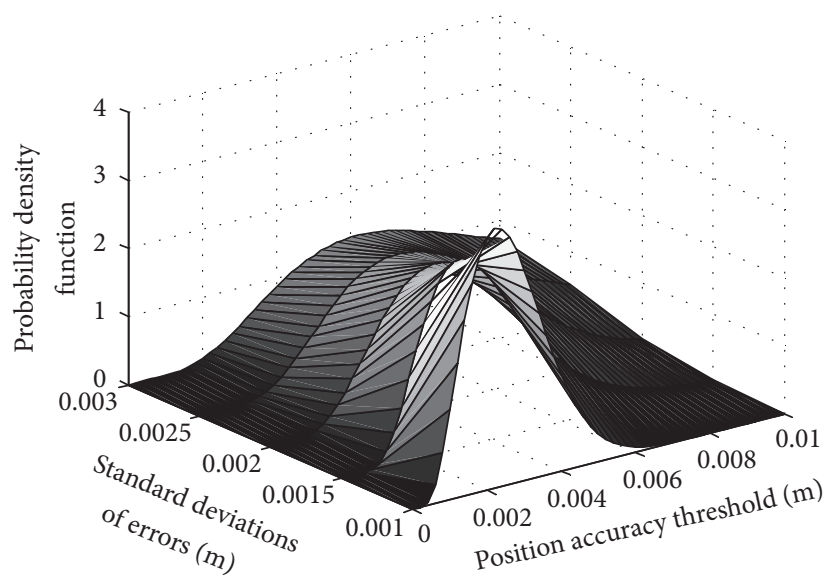

(a) PDF

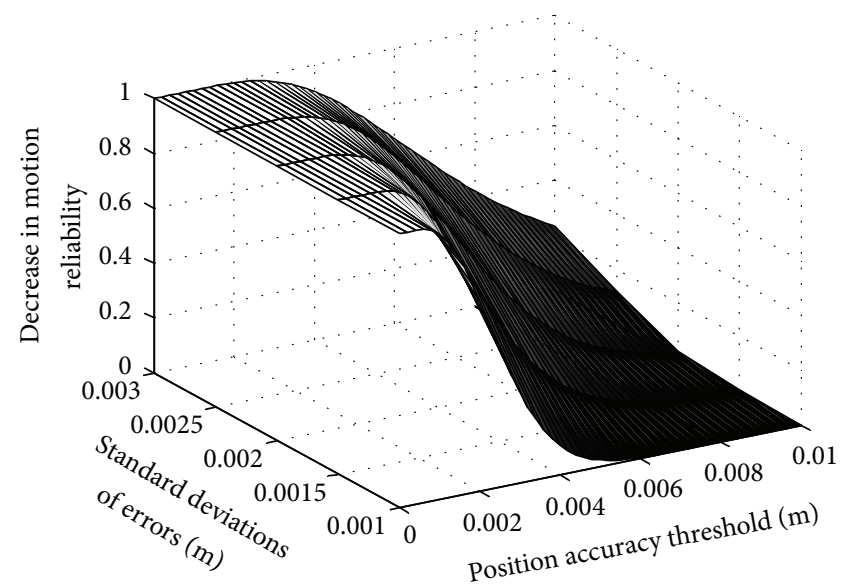

(b) Decrease in motion reliability

FIGURE 9: PDF and decease in motion reliability caused by different standard deviations and accuracy thresholds.

curves are achieved with the changes of position accuracy thresholds, which are shown in Figure 10. With the increase of errors, the motion reliability decreases obviously at the same accuracy threshold. At accuracy threshold $0.005 \mathrm{~m}$, when $\sigma$ increases from $0.015^{\circ}$ to $0.02^{\circ}$, the motion reliability decreases $17.65 \%$. Thus, the motion reliability can be improved signally by reducing errors.

Because of the time-varying characteristics of gear clearance, transmission error, and friction, the motion errors at different joint angles are different, which can be established as $e(\boldsymbol{\theta})$. Generally, every joint is limited into a certain range, which is determined by the characteristics of joint motor and configuration requirement of manipulator. For redundant manipulator, infinite feasible trajectories exist between $A$ and $B$. When position accuracy is guaranteed well in the entire trajectory, the motion reliability is high. Considering function $e(\boldsymbol{\theta})$, a proper trajectory should be chosen to arrange the joint angle at the range with smaller introduction of errors. Thus, strategy aiming at maximizing motion reliability can be described as follows.

Task constraints should be fulfilled firstly; namely, the position accuracy should be guaranteed in the entire trajectory, which can be expressed as $\mathbf{h}_{i}$. Then threshold constraints of parameters should be fulfilled during task, such as joint velocity and acceleration, which have the expression of $\mathbf{g}_{j}$. The objective function is established as the motion reliability of the trajectory tracking task. The output is motion reliability; the input is the joint angle range related to the selected 


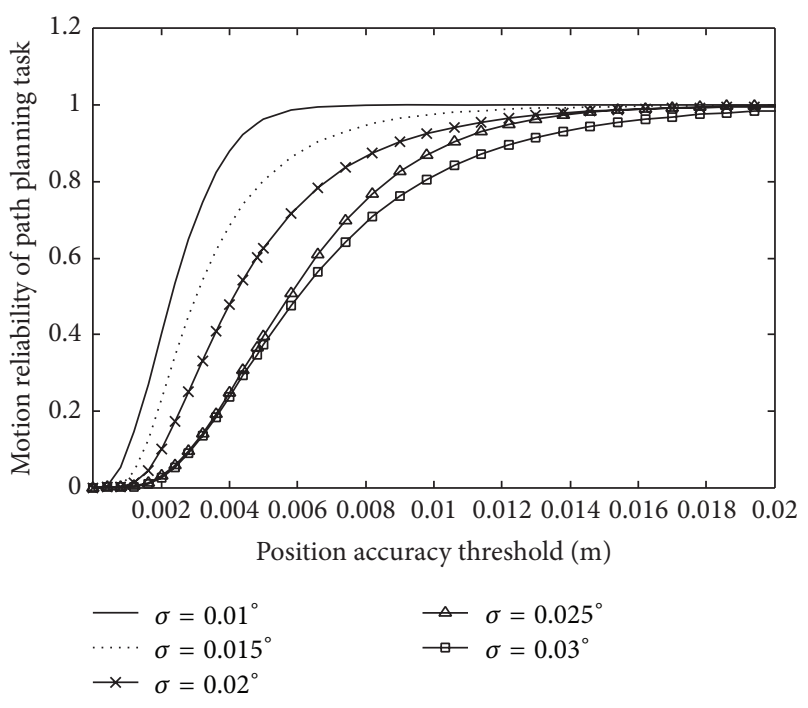

Figure 10: The motion reliability varies with errors of different standard deviation.

trajectory. Then the expression of the trajectory optimization can be concluded:

$$
\begin{array}{ll}
\text { find } & \text { Trajectory } A B \\
\max & f=\int\left(\cdots\left(\int p\left(x_{1}, \ldots, x_{s}\right) d x_{1}\right) \ldots\right) d x_{s} \\
\text { s.t. } & \mathbf{g}_{i} \leq 0, \mathbf{h}_{j}=0 \quad(i, j=1,2, \ldots)
\end{array}
$$

In trajectory $A B$, the corresponding range for joint $i$ is arranged as $\theta_{i} \in\left[\theta_{i}^{\min }, \theta_{i}^{\max }\right]$, during which the introduced joint angel errors are minimum. The objective function represents the motion reliability of the task, during which $p\left(x_{1}, \ldots, x_{s}\right)$ represents the PDF related to factors $x_{1}, \ldots, x_{s}$.

\section{Conclusion}

Motion reliability as a criterion can reflect the motion performance of manipulator synthetically. With the modeling and evaluation of motion reliability, the status of systems can be obtained and monitored in numerical during task operation. This paper establishes a universal model for motion reliability of a particular path planning task. The model which takes the factors related to position accuracy (such as clearance, wear, and friction) into account can reflect the contribution of these factors to motion reliability. Meanwhile, motion reliability can be evaluated by calculating the model. For model calculation, a model simplification method based on the approach of the equivalent extreme value is introduced. With this method, the multidimensional integral for PDF can be converted into one dimensional integral for PDF. Further, a method based on the maximum entropy principle is proposed to obtain the PDF. This method needs less samples and computation cost than the traditional Monte Carlo method, which makes it successful to achieve motion reliability evaluation with high precision and efficiency.
In the paper, the authors mean to establish a motion reliability model which can reflect the influence of factors related to position accuracy, such as clearance, wear, and friction,. Since it is difficult to derive the relationship between these factors and position accuracy, kinematics parameters are considered to be the intermediate variables to deliver the influence of the factors. Thus, the relationship between deviations of kinematics reliability and position accuracy is derived, based on which the model is established. However, the relationships between factors and kinematics parameters are not discussed in the paper, which should be devoted to further research.

From the model and motion reliability evaluation results, the contribution of each kinematics parameter to motion reliability can be concluded. According to the contribution, the importance of each kinematics parameter in improving motion reliability can be decided. When improving the motion reliability of manipulator, factors with high contribution should be given priority to handle. For example, in Section 4.4, because the joint angle contributes more than other parameters, the errors should be decreased firstly in operations. According to the motion reliability model and evaluation result, trajectory optimization or control strategy aiming at improving motion reliability of manipulator is a new optimization problem, which is meaningful for further research.

\section{Conflict of Interests}

The authors declare that there is no conflict of interests regarding the publication of this paper.

\section{Acknowledgments}

This work is supported by the National Key Basic Research Program of China (2013CB733000), the National Natural Science Foundation of China (61175080), and Specialized Research Fund for the Doctoral Program of Higher Education (20120005120004).

\section{References}

[1] B. S. Dhillon, A. R. M. Fashandi, and K. L. Liu, "Robot systems reliability and safety: a review," Journal of Quality in Maintenance Engineering, vol. 8, no. 3, pp. 170-212, 2002.

[2] J. Xia, Z. N. Jiang, H. Liu, H. Cai, and G. Wu, "A novel hybrid safety-control strategy for a manipulator," International Journal of Advanced Robotic Systems, vol. 11, no. 1, pp. 58-67, 2014.

[3] S. Rubrecht, V. Padois, P. Bidaud, M. de Broissia, and M. da Silva Simoes, "Motion safety and constraints compatibility for multibody robots," Autonomous Robots, vol. 32, no. 3, pp. 333349, 2012.

[4] J. Xia, Z. Jiang, H. Liu, H. Cai, and G. Wu, "A manipulator's safety control strategy based on fast continuous collision detection," in Proceedings of the IEEE International Conference on Robotics and Biomimetics (ROBIO '13), pp. 2380-2385, IEEE, December 2013.

[5] C. Zhang and G. Bai, "Reliability analysis of a two-link flexible robot manipulator," in Proceedings of the 2nd International 
Conference on Information Science and Engineering (ICISE '10), pp. 2269-2272, Hangzhou, China, December 2010.

[6] X. Huang and Y. Zhang, "Probabilistic approach to system reliability of mechanism with correlated failure models," Mathematical Problems in Engineering, vol. 2012, Article ID 465853, 11 pages, 2012.

[7] C. Carreras and I. D. Walker, "Interval methods for improved robot reliability estimation," in Proceedings of the Annual Reliability and Maintainability Symposium, pp. 22-27, January 2000.

[8] J. Li, J.-B. Chen, and W.-L. Fan, "The equivalent extremevalue event and evaluation of the structural system reliability," Structural Safety, vol. 29, no. 2, pp. 112-131, 2007.

[9] E. Taufer, S. Bose, and A. Tagliani, "Optimal predictive densities and fractional moments," Applied Stochastic Models in Business and Industry, vol. 25, no. 1, pp. 57-71, 2009.

[10] C. T. J. Xun, C. Zhang, and Y. Jiang, Electrical System Reliability Engineering, Science Press, Beijing, China, 2010.

[11] H. Zhuang, L. K. Wang, and Z. S. Roth, "Error-model-based robot calibration using a modified CPC model," Robotics and Computer Integrated Manufacturing, vol. 10, no. 4, pp. 287-299, 1993.

[12] G. Chen, Q. Jia, T. Li, and H. Sun, "Calibration method and experiments of robot kinematics parameters based on error model," Jiqiren/Robot, vol. 34, no. 6, pp. 680-688, 2012.

[13] W. Wu and S. S. Rao, "Uncertainty analysis and allocation of joint tolerances in robot manipulators based on interval analysis," Reliability Engineering \& System Safety, vol. 92, no. 1, pp. 54-64, 2007.

[14] W. Wu and S. S. Rao, "Interval approach for the modeling of tolerances and clearances in mechanism analysis," Journal of Mechanical Design, Transactions of the ASME, vol. 126, no. 4, pp. 581-592, 2004.

[15] M. D. Pandey and X. Zhang, "System reliability analysis of the robotic manipulator with random joint clearances," Mechanism and Machine Theory, vol. 58, pp. 137-152, 2012.

[16] S. S. Rao and P. K. Bhatti, "Probabilistic approach to manipulator kinematics and dynamics," Reliability Engineering and System Safety, vol. 72, no. 1, pp. 47-58, 2001.

[17] H. P. Jawale and H. T. Thorat, "Positional error estimation in serial link manipulator under joint clearances and backlash," Journal of Mechanisms and Robotics, vol. 5, no. 2, Article ID 021003, 2013.

[18] H. Abdellatif and B. Heimann, "On compensation of passive joint friction in robotic manipulators: Modeling, detection and identification," in Proceedings of the Computer Aided Control System Design, IEEE International Conference on Control Applications, IEEE International Symposium on Intelligent Control, pp. 2510-2515, Munich, Germany, October 2006.

[19] Z. F. Bai, Y. Zhao, and J. Chen, "Dynamics analysis of planar mechanical system considering revolute clearance joint wear," Tribology International, vol. 64, pp. 85-95, 2013.

[20] J. Kim, W.-J. Song, and B.-S. Kang, "Stochastic approach to kinematic reliability of open-loop mechanism with dimensional tolerance," Applied Mathematical Modelling, vol. 34, no. 5, pp. 1225-1237, 2010.

[21] N. Kumar, J.-H. Borm, and A. Kumar, "Reliability analysis of waste clean-up manipulator using genetic algorithms and fuzzy methodology," Computers \& Operations Research, vol. 39, no. 2, pp. 310-319, 2012.
[22] S. P. Sharma, D. Kumar, and A. Kumar, "Reliability analysis of complex multi-robotic system using GA and fuzzy methodology," Applied Soft Computing Journal, vol. 12, no. 1, pp. 405-415, 2012.

[23] L. Dashuang, W. Kesheng, Z. Longlong et al., "Dynamic precision reliability analysis for six degrees of freedom microdisplacement mechanism of reticle stage in lithography machine based on Monte-Carlo," in Proceedings of the International Conference on Quality, Reliability, Risk, Maintenance, and Safety Engineering (QR2MSE '13), pp. 250-252, 2013.

[24] T. Li, Q. X. Jia, G. Chen et al., "Motion reliability assessment of robot for trajectory tracking task," Systems Engineering and Electronics, vol. 36, no. 12, pp. 2556-2561, 2014.

[25] P. L. Novi Inverardi and A. Tagliani, "Maximum entropy density estimation from fractional moments," Communications in Statistics. Theory and Methods, vol. 32, no. 2, pp. 327-345, 2003.

[26] G. Chen, L. Zhang, Q. X. Jia, M. Chu, and H. Sun, "Repetitive motion planning of free-floating space manipulators," International Journal of Advanced Robotic Systems, vol. 10, pp. 253-263, 2013.

[27] E. Jaynes, "Review of 'principles of statistical mechanics-the information theory approach' (Katz, A.; 1967)," IEEE Transactions on Information Theory, vol. 14, no. 4, pp. 611-612, 1968.

[28] X. Li, The Method Study about Probability Distribution Based on the Principle of Maximum Entropy, North China Electric Power University, Beijing, China, 2008. 


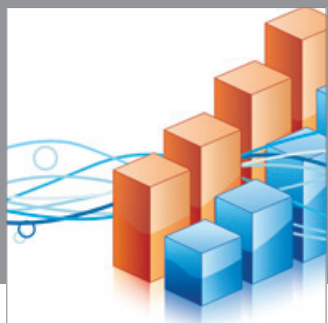

Advances in

Operations Research

mansans

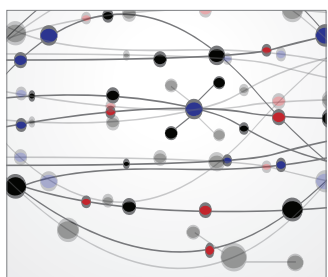

The Scientific World Journal
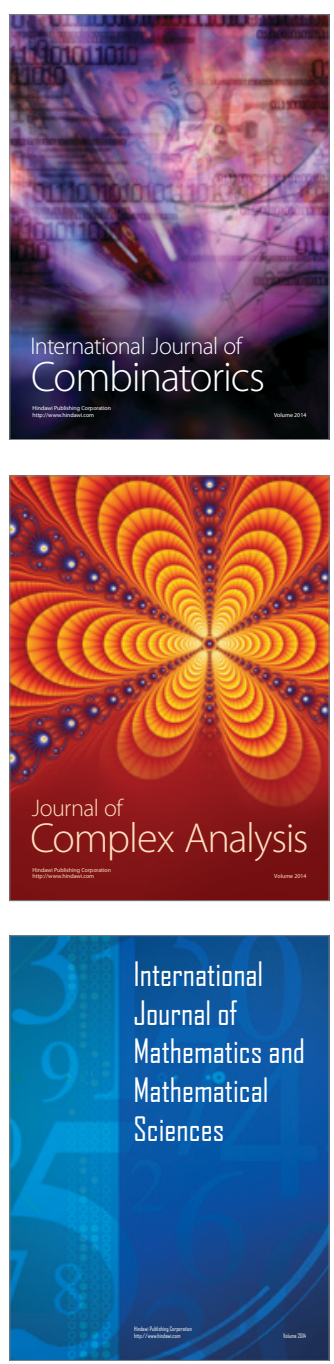
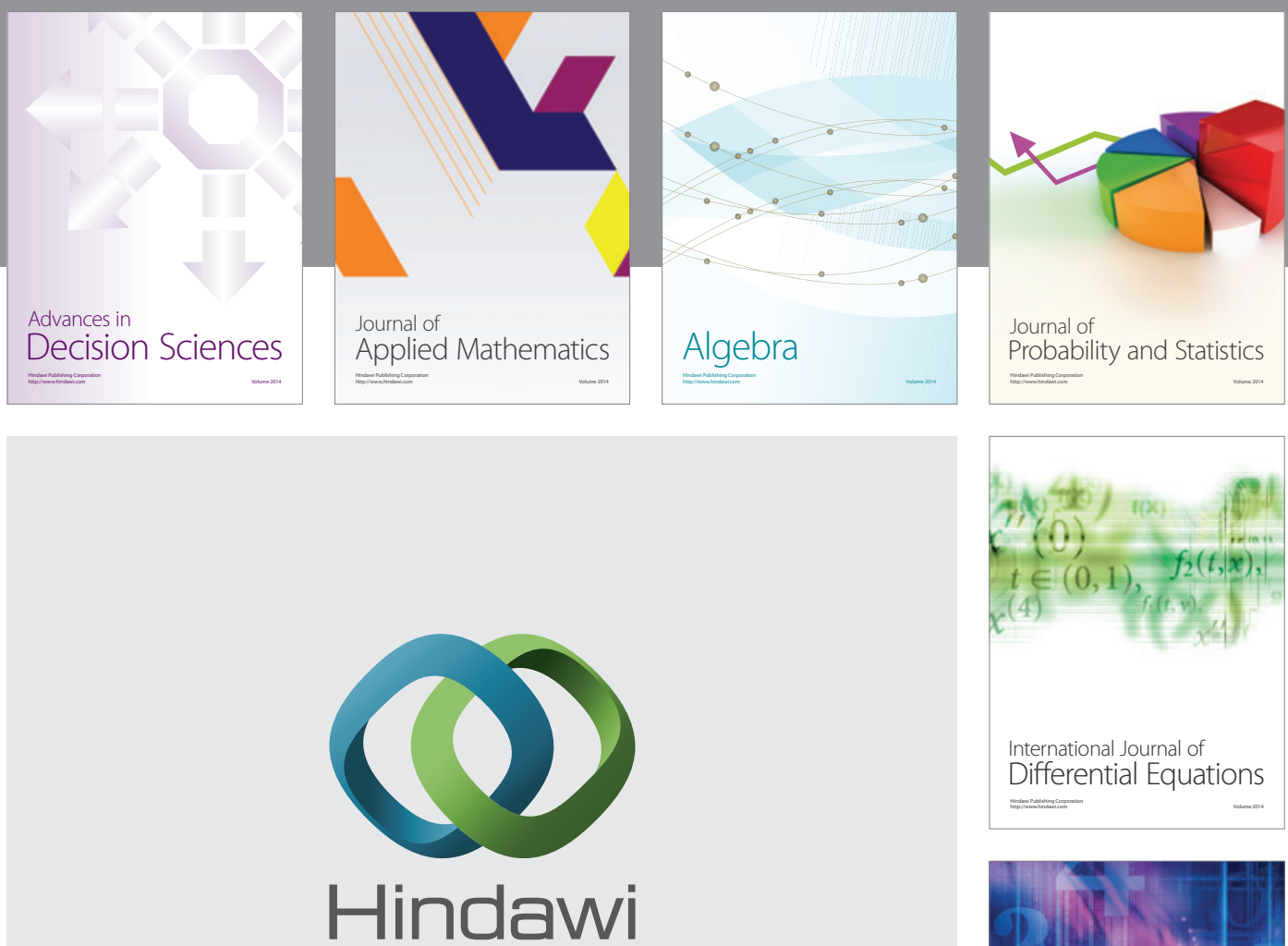

Submit your manuscripts at http://www.hindawi.com
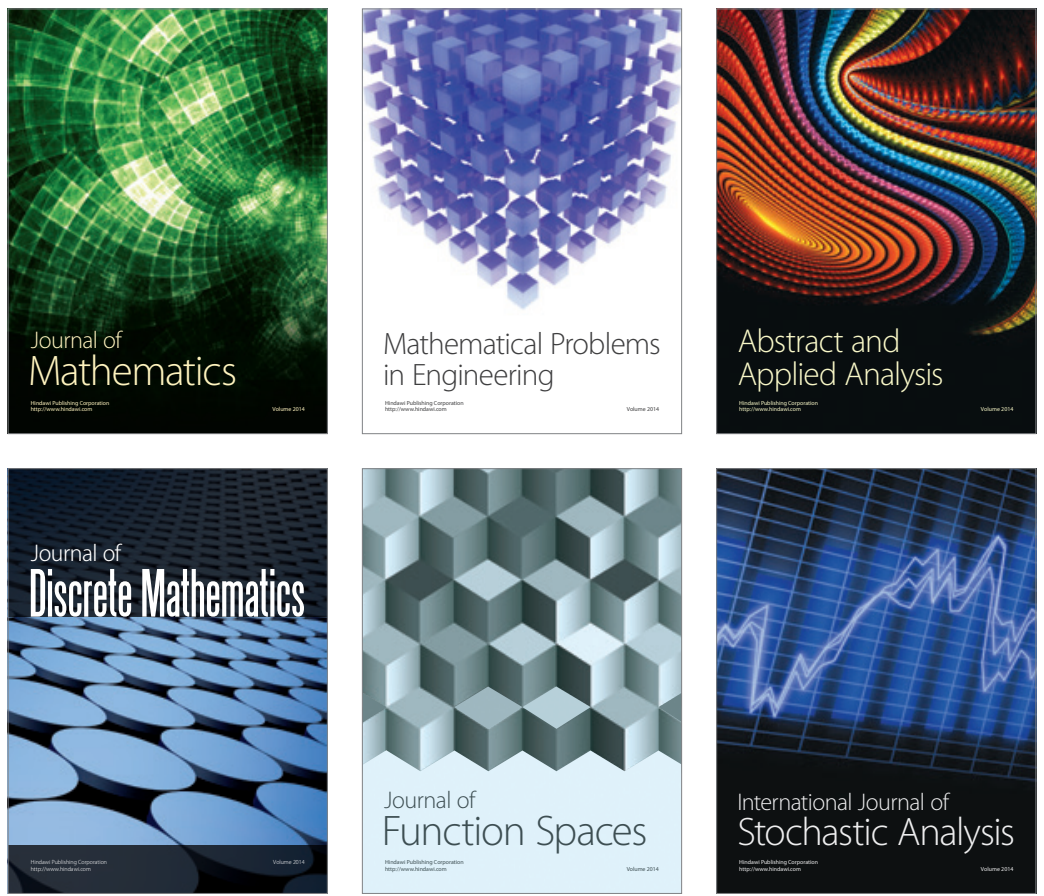

Journal of

Function Spaces

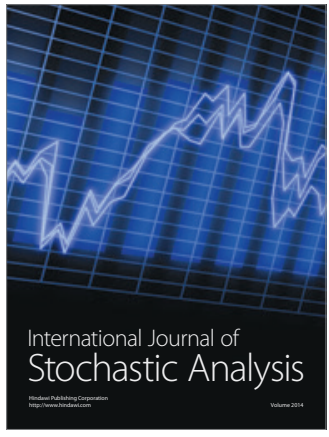

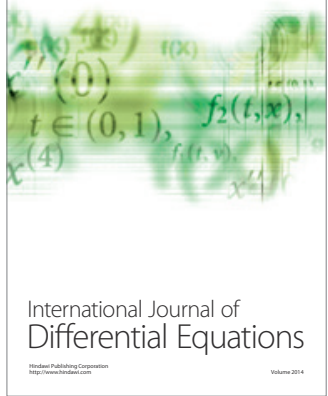
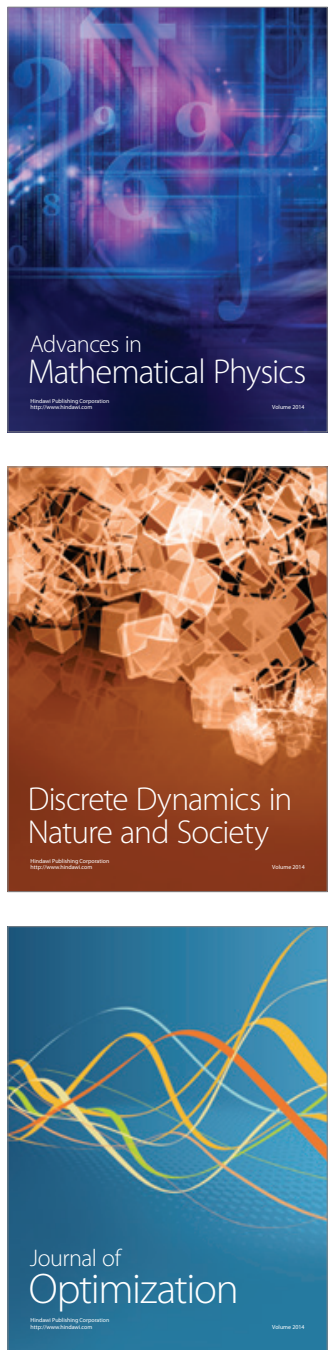